Investigating the Relationship Between QoS and QoE in a Mixed Desktop/Handheld Gaming Setting

Peer-reviewed author version

WIJNANTS, Maarten; AGTEN, Stijn; QUAX, Peter \& LAMOTTE, Wim (2009) Investigating the Relationship Between QoS and QoE in a Mixed Desktop/Handheld Gaming Setting. In: Proceedings of CONEXT. p. 29-30..

Handle: http://hdl.handle.net/1942/10327 


\title{
Investigating the Relationship Between QoS and QoE in a Mixed Desktop/Handheld Gaming Setting
}

\author{
Maarten Wijnants Stijn Agten Peter Quax Wim Lamotte \\ Hasselt University / tUL / IBBT / Expertise Centre for Digital Media \\ Wetenschapspark 2, 3590 Diepenbeek, Belgium \\ \{maarten.wijnants,stijn.agten,peter.quax,wim.lamotte\}@uhasselt.be
}

\begin{abstract}
Innovative software and system development requires a multidisciplinary approach where technical, objectively quantifiable Quality of Service aspects are integrated with and correlated to subjective Quality of Experience measures such as usability, user expectations and experiences. To date, QoS and QoE research has however mainly been carried out in an isolated manner. Their exact correlation consequently remains unclear. Using a collaborative multi-platform game as case study, we intend to investigate this relationship and hope to bridge the divide that currently exists between social, user-oriented research and technically-oriented application development by formulating recommendations and guidelines. To implement the QoS and QoE optimizations, we will rely on the NIProxy, a network proxy equipped with network traffic shaping and service provision functionality.
\end{abstract}

\section{Categories and Subject Descriptors}

C.2.1 [Computer Systems Organization]: ComputerCommunication Networks - network architecture and design

\section{General Terms}

Experimentation, Human Factors, Measurement, Performance

\section{Keywords}

QoS, QoE, NIProxy, Multi-Platform Gaming

\section{INTRODUCTION}

Quality of Service (QoS) is a well-established concept in software and system engineering. It is a measure of technological excellence as it denotes the capability of systems to guarantee that a certain level of performance will be met. Quality of Experience (QoE) on the other hand deals with user expectation, satisfaction and overall experience. Compared to QoS, it is a subjective metric that involves human dimensions and that is hence not easily quantified or objectively measured. A common approach to obtain QoE feedback is through qualitative user studies.

Despite their different perspective, QoS and QoE are unquestionably related. The level of service provided by an application or software system is sure to influence the user

Copyright is held by the author/owner(s).

CoNEXT Student Workshop'09, December 1, 2009, Rome, Italy. ACM 978-1-60558-751-6/09/12. satisfaction. On the other hand, a high QoS level not necessarily yields an excellent QoE. As a result, there is a need to thoroughly chart the relationship between both concepts. To date however, QoS/QoE research has largely been carried out in a fragmented manner, focusing on measuring and optimizing either objective technical QoS parameters or subjective QoE dimensions. In the former category, [1] for instance presents a methodology for devising a cross-layer performance-energy trade-off, however without considering the user satisfaction. An example in the latter category is the study described in [2], which relates video quality to user experience in the context of a video conferencing system, without linking the findings to objective QoS factors that can be applied to network services. Our goal is to bridge the QoS/QoE gap and to uncover the correlation between both concepts.

\section{QoS/QoE RESEARCH IN A HETEROGE- NEOUS GAMING ENVIRONMENT}

To carry out our research, we have selected on-line computer gaming as target application domain. Networked gaming is a suitable setting to perform QoS/QoE studies as it imposes numerous QoS- and QoE-related requirements that are likely to have a significant impact on the user experience. Examples include the distribution of real-time game data, the visualization of game-related data on the enduser device, (audiovisual) communication capabilities between players and efficient user interaction mechanisms. To further increase the number of possible QoS/QoE links, a collaborative multi-platform gaming scenario is envisioned where desktop and mobile players need to cooperate to accomplish shared objectives. Besides heterogeneity in the end-user device space, the multi-platform setting also introduces variability in connectivity and network performance (i.e. wired versus wireless network connections). For the handheld devices, we have opted to use smartphones running Google's Android OS.

\section{METHODOLOGY}

Within this gaming context, we plan to monitor and optimize QoS as well as QoE parameters and to investigate their correlation and the way they influence each other. QoS measurement and optimization will take place on both network and device level. On the network level, we will deploy probes to deduce network capacity and performance (in terms of throughput, error rate, latency, ...), perform network traffic shaping and bandwidth brokering to ensure 


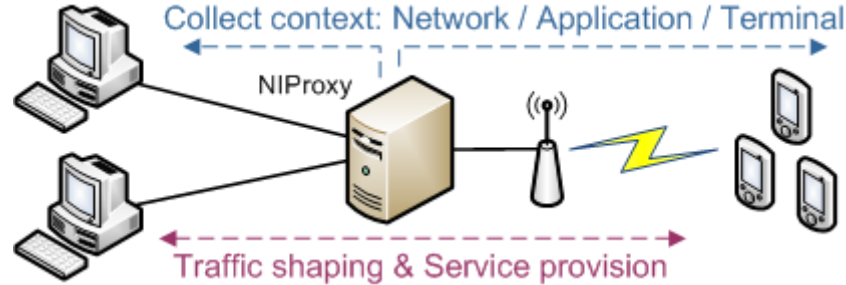

Figure 1: NIProxy deployment in the targeted desktop/handheld gaming setting.

optimal use of the available capacity, deliberately adapt network traffic during dissemination, and so on. Regarding device QoS parameters, factors that will be considered include device capabilities and features (such as audiovisual codec support) and performance-related statistics (achieved frame rate, battery lifetime, CPU utilization, etcetera). Concerning QoE, we will evaluate mechanisms which allow users to specify their preferences as well as other contextual information (e.g. environmental factors) and we will examine the ability to determine such information implicitly (e.g. automated user profiling). To obtain QoE-related data, we plan to organize qualitative user studies in a living lab test environment that is representative for the real-life setting in which the application is to be used. The goal of these studies will be to determine user expectations (e.g. by interviewing users before the gaming session is started), to attempt to quantify user satisfaction (e.g. through observation during play) and to collect user feedback (for instance through a questionnaire or post-gaming interview).

Based on the data collected in this particular gaming context, we will attempt to identify the mutual influence QoS and QoE parameters have on each other and we will try to translate our findings into a set of recommendations and guidelines for designers and developers.

\section{NETWORK INTELLIGENCE PROXY}

A central role in our QoS/QoE research will be reserved for the Network Intelligence Proxy (NIProxy, see figure 1) [3]. The NIProxy is a network intermediary which attempts to optimize the experience of users of distributed applications by introducing different types of intelligence in the network infrastructure. The NIProxy's contextual knowledge is threefold and includes information regarding the networking substrate, the application and the user's terminal. This intelligence is exerted to implement two distinct yet interoperable user experience optimization techniques, namely network traffic shaping and multimedia service provision. In the former technique, the NIProxy's gathered intelligence is exploited to manage the bandwidth consumption of distributed applications and the way bandwidth is distributed among involved network flows. The service provision mechanism on the other hand enables the NIProxy to in-network process data that is exchanged as part of distributed applications.

We expect the NIProxy's optimization mechanisms will enable adequate response to fluctuations in QoS and QoE parameters in the envisioned gaming scenario. Its network traffic shaping functionality will for instance allow the NIProxy to react to variable network connection throughput by reshuffling network flow bandwidth assignment based on relative flow importance. By applying services on the other hand, the NIProxy will be able to streamline data dissemination and to mitigate mismatches between the capabilities of desktop and handheld devices. Anticipated services include the addition of FEC protection to data that needs to be transmitted over error-prone (i.e. wireless) network links, the transcoding of multimedia data to lower quality to reduce their bitrate in times of bandwidth shortage, the mixing of multiple audio streams into a single signal to reduce bandwidth consumption and to alleviate the load on the (handheld) terminal, content adaptation based on terminal capabilities and altering user preferences, combining multiple video streams into a mosaic, ans so on.

The influence of the decisions and actions taken by the NIProxy on the user satisfaction will be investigated through afore-mentioned living lab user studies. Based on the resulting QoE measurements and user feedback, the operation of the NIProxy will be adjusted where needed. This will be an iterative process, meaning the adjustments implemented based on previous QoE findings will be subjected to subsequent user studies.

\section{CURRENT STATUS AND NEXT STEPS}

We are currently in the process of implementing a multiplatform game that will serve as subject for our QoS/QoE research. One aspect we are paying particular attention to is inter-player (audiovisual) communication, as decent communication facilities are of paramount importance in a collaborative gaming setting. The NIProxy and its user experience optimization mechanisms have already been implemented and experimentally evaluated in multiple contexts. Readily available NIProxy services include video transcoding, a service to FEC-protect data and a video mosaic service. Shortterm action points include the further development of the multi-platform game and extending it with an Augmented Reality (AR) aspect, the implementation of additional NIProxy services, the preparation of the living lab test environment and the execution of initial user studies. In the more distant future, the outcome of these studies will be analyzed and translated into next implementational steps and objectives.

\section{ACKNOWLEDGMENTS}

This research is part of the IBBT ISBO Gr@sp project. Research at EDM is also partly funded by the EFRD.

\section{REFERENCES}

[1] W. Eberle, B. Bougard, S. Pollin, and F. Catthoor. From Myth to Methodology: Cross-Layer Design for Energy-Efficient Wireless Communication. In Proc. 42nd Annual Design Automation Conference, pages 303-308, Anaheim, California, USA, 2005.

[2] A. Watson and A. M. Sasse. Measuring Perceived Quality of Speech and Video in Multimedia Conferencing Applications. In Proc. 6th ACM International Conference on Multimedia, pages 55-60, Bristol, United Kingdom, 1998.

[3] M. Wijnants and W. Lamotte. Managing Client Bandwidth in the Presence of Both Real-Time and non Real-Time Network Traffic. In Proc. 3rd International Conference on COMmunication System softWAre and MiddlewaRE, Bangalore, India, January 2008. 